\title{
Pseudopotentials and symmetries of evolution equations
}

\author{
Kazuhiro KISO \\ (Received November 6, 1987, Revised June 28, 1988)
}

\section{§ 1. Introduction}

In the development of the soliton theory the symmetries of differential equations played an important role. The Lie-Bäcklund transformation was defined and investigated by M. K. Ibragimov and R. L. Anderson [1], P. T. Olver [5], A. M. Vinogradov and I. S. Krasil'shchik [6] and others. It is known that the soliton equations have infinitely many Lie-Bäcklund transformations.

It is also known that some soliton equations (for example, the $\mathrm{KdV}$ equation) have a operator $L$ called a recursion operator, which has the following properties : If $\phi$ is a Lie-Bäcklund transformation, then $L \phi$ is also a Lie-Bäcklund transformation. (See [5].) In general $L \phi$ can not be defined in the usual function space on the infinite-jet space, because $L$ contains terms involving the integrals of the unknown function. Besides this, some observations show that it is useful to extend the notion of the Lie-Bäcklund transformation. (See [6], [7].) For these reasons, A. M. Vinogradov and I. S. Krasil'shchik defined a non-local symmetry of differential equations which is a natural generalization of Lie-Bäcklund transformation. In this paper we consider their symmetry in case of the evolution equations from a slightly different view point.

Let us consider the evolution equation

$$
u_{t}=F\left(x, t, u, u_{1}, \ldots, u_{n}\right)
$$

where $u=u(x, t)$ and $u_{i}=\frac{\partial^{i} u}{\partial x^{i}}$. Considering $u_{i}$ as an independent variable, we denote by $R$ the infinite dimensional manifold having coordinates $(x, t, u$, $u_{1}, u_{2}, \ldots$ ). Let $\partial$ (resp. $\partial_{t}$ ) be the total derivative with respect to $x$ (resp. $t$ ). We have $\partial \partial_{t}=\partial_{t} \partial$. Set $A=C^{\infty}(R)$. (In $\S 5$ we consider only polynomial functions on $R$ by some technical reasons.) The triplet $S=\left\{A ; \partial, \partial_{t}\right\}$ is a geometric object corresponding to the differential equation (1.1). Then a Lie-Bäcklund transformation of (1.1) is a function $\phi$ contained in $A$ satisfying 


$$
\partial_{t} \phi=D_{\phi} F,
$$

where

$$
D_{\phi}=\sum_{i=0}^{\infty} \partial^{i} \phi \partial / \partial u_{i} .
$$

We can generalize these situation as follows. Introduce new variables $w_{1}, w_{2}, \ldots, w_{m}$ and set $A_{1}=C^{\infty}\left(R \times\left\{\left(w_{1}, w_{2}, \ldots, w_{m}\right)\right\}\right)$. Suppose that the derivations $\partial$ and $\partial_{t}$ of $A$ can be extended to derivations of $A_{1}$ which commute with each other. $w_{1}$ 's are nothing but the pseudopotentials in the sense of [8]. Suppose that a function $\phi \in A_{1}$ satisfies (1.2). This is equivalent to the condition that the derivation $D_{\phi}$ from $A$ to $A_{1}$ commutes with $\partial_{t}$. Then in $\S 4$ we prove that there exists an extended differential algebra $A_{2}$ of $A_{1}$ such that $D_{\phi}$ can be extended to a derivation $A_{1} \longrightarrow A_{2}$ commuting with $\partial$, $\partial_{t}$. (See Theorem 1.) Using this theorem repeatedly, we know that there exists an extended differential algebra $S_{\infty}=\left\{A_{\infty}: \partial, \partial_{t}\right)$ of $S$ such that $D_{\phi}$ can be extended to a derivation of $A_{\infty}$ commuting with $\partial, \partial_{t}$. This is the nonlocal symmetry in the sense of [7]. That is to say, Theorem 1 means that we can construct $S_{\infty}$ and a non-local symmetry from the symmetry $\phi$ satisfying (1.2).

On the other hand it is well known that the soliton equations have symmetries called Bäcklund transformations. A Bäcklund transformation is defined by partial differential equations and is not obtained by integrating Lie-Bäcklund transformations. In $\S 3$, we consider "the infinitesimal version " of Bäcklund transformations of the $\mathrm{KdV}$, or the modified $\mathrm{KdV}$ equations and show that they are symmetries in the above sense. This suggests that the differential equations having many non-local symmetries are "good" equations such as soliton equations.

Finally in $\S 5$ we construct $S_{\infty}$ in case of the $\mathrm{KdV}$ equation. We note that we used the algebraic computation system REDUCE3 for the cumbersome calculations in this section.

\section{§2. Lie-Bäcklund transformations}

Let us consider the evolution equation (1.1). We use the same notations as in $\S 1$. Therefore $R$ denotes the infinite dimensional manifold having coordinates $\left(x, t, u, u_{1}, u_{2}, \ldots\right)$ and $A=C^{\infty}(R)$. Let $\partial$ (resp. $\left.\partial_{t}\right)$ be the total derivative with respect to $x$ (resp. $t$ ), i. e.,

$$
\partial=\partial / \partial x+\sum_{i=0}^{\infty} u_{i+1} \partial / \partial u_{i}
$$

and

$$
\partial_{t}=\partial / \partial t+\sum_{i=0}^{\infty} \partial^{i} F \partial / \partial u_{i} .
$$


$\partial$ and $\partial_{t}$ are derivations of $A$ which commute with each other.

A function $\phi \in A$ satisfying (1.2) is called a Lie-Bäcklund transformation (LBT) of (1.1). Note that (1.2) is equivalent to the condition $\partial_{t} D_{\phi}=$ $D_{\phi} \partial_{t}$.

EXAMPLE. The KdV equation : $u_{t}=u_{3}+12 u u_{1}$

Let $L=\partial^{2}+8 u+4 u_{1} \partial^{-1}$. It is known that if $\phi$ is a LBT of (2.1), then $L \phi$ (if defined) is also a LBT. $L$ is called a recursion operator. (See [5].) Obviously $u_{1}$ is a LBT, and so we have an infinite series $\phi_{k}=L^{k} u_{1}$ of LBT's. The first two of these are

$$
\begin{aligned}
& \phi_{1}=u_{3}+12 u u_{1}, \\
& \phi_{2}=u_{5}+40 u_{1} u_{2}+20 u u_{3}+120 u^{2} u_{1} .
\end{aligned}
$$

The evolution equations $u_{t}=\phi_{k}$ are nothing but the higher order KdV equations. Besides the above series there exist two LBT's :

$$
\begin{aligned}
& \tilde{\phi}_{1}=t u_{1}+1 / 12, \\
& \tilde{\phi}=t u_{3}+x u_{1} / 3+12 t u u_{1}+2 u / 3 .
\end{aligned}
$$

(See [6].) Note that $L \tilde{\phi}_{2}$ is not defined in $A$.

\section{$\S 3$. Symmetry}

In this section, we consider a generalization of the Lie-Bäcklund transformation. Let $S=\left\{A ; \partial, \partial_{t}\right\}$ be as in the previous sections. Let $w_{1}$, $w_{2}, \ldots, w_{m}$ be new independent variables. Set $R_{1}=R \times\left\{\left(w_{1}, w_{2}, \ldots, w_{m}\right)\right\}$ and $A_{1}=C^{\infty}\left(R_{1}\right)$. Suppose that the derivations $\partial, \partial_{t}$ of $A$ can be extended to derivations of $A_{1}$ such that $\partial \partial_{t}=\partial_{t} \partial$. For brevity, we use the same natations $\partial, \partial_{t}$ for the corresponding derivations in $A_{1}$. We call the differential algebra $S=\left\{A ; \partial, \partial_{t}\right\}$ an extended differential algebra of $S$. $w_{i}$ 's are called the pseudopotentials constituting $S_{1}$. As before, for $\phi \in A_{1}, D_{\phi}$ denotes the derivation from $A$ to $A_{1}$ defined by (1.3).

DEfinition. $\phi$ is called a symmetry of (1.1) if and only if the following equation holds :

$$
\partial_{t} \phi=D_{\phi} F \text {. }
$$

Note that (3.1) is equivalent to the condition $\partial_{t} D_{\phi}=D_{\phi} \partial_{t}$.

EXAMPLE 1. The modified KdV equation:

$$
u_{t}=u_{3}+6 u^{2} u_{1} \text {. }
$$

Define a potential $w$ by 


$$
\left\{\begin{array}{l}
w_{x}=u \\
w_{t}=u_{2}+2 u^{3}
\end{array}\right.
$$

Let $\widetilde{w}$ be the solution of the following differential equation:

$$
\begin{aligned}
& \left\{\begin{array}{l}
(\widetilde{w}-w)_{x}=k \sin (\widetilde{w}+w) \\
(\widetilde{w}-w)_{t}=k\left\{\left(\widetilde{w}_{x \dot{x}}+w_{x x}\right) \cos (\widetilde{w}+w)\right.
\end{array}\right. \\
& \left.+\left(\widetilde{w}_{x}^{2}+w_{x}^{2}\right) \sin (\widetilde{w}+w)\right\},
\end{aligned}
$$

where $k$ is an arbitrary constant. Then, if $u$ is a solution of (3.2), $\tilde{u}=\widetilde{w}_{x}$ is also a solution of (3.2). The transformation $u \longrightarrow \tilde{u}$ is called a Bäcklund transformation of (3.2). Note that $\widetilde{w}$ depends on $k$ and the transformation $u \longrightarrow \tilde{u}$ is a l-parameter family of transformations. Although it is not a transformation in the strict sense, we can consider its infinitesimal version as follows. Assuming that $\widetilde{w}=w$ when $k=0$, we set $W=\left.\frac{\partial \widetilde{w}}{\partial k}\right|_{k=0}$. Then differentiating (3.3) with respect to $k$ and setting $k=0$, we get

$$
\left\{\begin{array}{l}
W_{x}=\sin 2 w \\
W_{t}=2 w_{x x} \cos 2 w+2 w_{x}^{2} \sin 2 w .
\end{array}\right.
$$

By the definition of $W$ we can say that $\phi=\sin 2 w$ is " the infinitesimal transformation" of (3.3). The significance of (3.4 b) will be clear in the next section. The infinitesimal transformation $\phi$ can be interpreted as follows. Let $R_{1}=R \times\{w\}$ and $A_{1}=C^{\infty}\left(R_{1}\right)$. Extend the derivations $\partial$ and $\partial_{t}$ of $A$ to the derivations of $A_{1}$ by

$$
\left\{\begin{array}{l}
\partial w=u \\
\partial_{t} w=u_{2}+2 u^{3}
\end{array}\right.
$$

It is easy to see that $\partial \partial_{t}=\partial_{t} \partial$ holds in $A_{1}$. Now define the derivation $D_{\phi}$ : $A \longrightarrow A_{1}$ by (1.3). Then it is clear that $D_{\phi} \partial=\partial D_{\phi}$, and moreover we can show by direct calculations that $D_{\phi} \partial_{t}=\partial_{t} D_{\phi}$. That is to say, $\phi$ is a symmetry in the sense of the above definition.

EXAMPLE 2. The KdV equation

In this case the Bäcklund transformation can be stated as follows. (See [8].) Define a pseudopotential $y$ by

$$
\left\{\begin{array}{l}
y_{x}=2 u+y^{2}-k \\
y_{t}=4(u+k)\left(2 u+y^{2}-k\right)+2 u_{2}+4 u_{1} y .
\end{array}\right.
$$

Then, if $u$ is a solution of (2.1), $\tilde{u}=-u-y^{2}+k$ is also a solution of (2.1). Although the following arguments are quite formal and heuristic, we can consider the infinitesimal version as follows. First of all, to declare the 
dependence of $y$ (resp. $\tilde{u})$ on $k$ let us write $y(k)$ (resp. $\tilde{u}(k))$ instead of $y$ (resp. $\tilde{u})$. We denote by $T(k)$ the transformation $u \longrightarrow \tilde{u}(k)$. Note that $T(0)$ is not the identity transformation, so that $\left.\frac{\partial T(k)}{\partial k}\right|_{k=0}$ is not an infinitesimal transformation. Therefore, fixing $k_{0}$, we set $T\left(k_{0}\right)^{-1}(\tilde{u}(k))=$ $U(k)$. We have

$$
\tilde{u}(k)=-U(k)-Y(k)^{2}+k_{0},
$$

where $Y(k)$ is the pseudopotential defined by

$$
\left\{\begin{array}{r}
Y(k)_{x}=2 U(k)+Y(k)^{2}-k_{0} \\
Y(k)_{t}=4\left(U(k)+k_{0}\right)\left(2 U(k)+Y(K)^{2}-k_{0}\right) \\
+2 U(k)_{x x}+4 U(k)_{x} Y(k) .
\end{array}\right.
$$

Hence we have

$$
\begin{aligned}
U(k) & =-\tilde{u}(k)-Y(k)^{2}+k_{0} \\
& =u+y(k)^{2}-k-Y(k)^{2}+k_{0} .
\end{aligned}
$$

Set $\quad \phi=\left.\frac{\partial U(k)}{\partial k}\right|_{k=k_{0}}, w=y\left(k_{0}\right), w^{\prime}=\left.\frac{\partial y(k)}{\partial k}\right|_{k=k_{0}}$ and $\quad v=\left.\frac{\partial Y(k)}{\partial k}\right|_{k=k_{0}}$. Differentiating (3.8) with respect to $k$ and then setting $k=k_{0}$, we get

$$
\psi=2 w w^{\prime}-1-2 w v,
$$

where we assumed that $U\left(k_{0}\right)=u$ and $Y\left(k_{0}\right)=y\left(k_{0}\right)=w$. Since $k_{0}$ is arbitrary, let us write $k$ instead of $k_{0}$. Note that $w^{\prime}=\frac{\partial w}{\partial k}$ and $v$ are the pseudopotentials defined by

$$
\left\{\begin{array}{l}
w_{x}^{\prime}=2 w w^{\prime}-1 \\
w_{t}^{\prime}=4\left(2 u+w^{2}-k\right)+4(u+k)\left(2 w w^{\prime}-1\right)+4 u_{1} w^{\prime}
\end{array}\right.
$$

and

$$
\left\{\begin{array}{r}
v_{x}=2\left(2 w w^{\prime}-1-w v\right) \\
v_{t}=4\left(-u_{1} v+2 u_{1} w^{\prime}-2 u v w+4 u w^{\prime} w\right. \\
\left.-2 k v w+4 k w^{\prime} w+w^{2}-3 k\right) .
\end{array}\right.
$$

As in the case of the modified $\mathrm{KdV}$ equation, let us extend the differential algebra $S=\left\{A ; \partial, \partial_{t}\right\}$ as follows. Set $R_{1}=R \times\left\{\left(w, w^{\prime}, v\right)\right\}$ and $A_{1}=C^{\infty}\left(R_{1}\right)$. Extend the derivations $\partial$, $\partial_{t}$ to the derivations of $A_{1}$ by using the right hand sides of the equations (3.6), (3.10) and (3.11). For example, $\partial w^{\prime}=2 w w^{\prime}-1$ and $\partial v=2\left(2 w w^{\prime}-1-w v\right)$. Then it is easy to see that $\partial \partial_{t}=\partial_{t} \partial$. Moreover we can prove by direct calculations that the derivation $D_{\psi}: A \longrightarrow A_{1}$ defined by (1.3) commutes with $\partial_{t}$. 


\section{$\S 4$. Extension of derivation}

Let $R$ and $S$ be as in the previous sections. Let $S_{1}=\left\{A_{1} ; \partial, \partial_{t}\right\}$ be an extended differential algebra of $S$ and $\phi \in A_{1}$ be a symmetry. Let $w_{1}$, $w_{2}, \ldots, w_{m}$ be the pseudopotentials constituting $S_{1}$. Let $z_{1}, z_{2}, \ldots, z_{l}$ be new independent variables. Set $R_{2}=R_{1} \times\left\{\left(z_{1}, z_{2}, \ldots, z_{l}\right)\right\}$ and $A_{2}=C^{\infty}\left(R_{2}\right)$. Suppose that the derivations $\partial, \partial_{t}$ of $A_{1}$ can be extended to commutative derivations of $A_{2}$. Then we say that $S_{2}=\left\{A_{2} ; \partial, \partial_{t}\right\}$ is an extended differential algebra of $S_{1}$. We can prove

THEOREM 1. There exists an extended differential algebra $S_{2}=\left\{A_{2} ; \partial\right.$, $\left.\partial_{t}\right\}$ of $S_{1}$ such that $D_{\phi}$ can be extended to a derivation from $A_{1}$ to $A_{2}$ commuting with $\partial, \partial_{t}$.

PROOF. Set $\partial w_{\alpha}=f_{\alpha}$ and $\partial_{t} w_{\alpha}=g_{\alpha}$, where $f_{\alpha}, g_{\alpha} \in A_{1}$. We have $\partial_{t} f_{\alpha}=$ $\partial g_{\alpha}$. Suppose that there exists $S_{2}$. Set $D_{\phi} w_{\alpha}=z_{\alpha}$. Then the commutativity of $D_{\phi}$ with $\partial$ and $\partial_{t}$ implies

$$
\begin{aligned}
\partial z_{\alpha} & =D_{\phi} f_{\alpha} \\
& =\sum \frac{\partial f_{\alpha}}{\partial u_{i}} \partial^{i} \phi+\sum \frac{\partial f_{\alpha}}{\partial w_{\beta}} z_{\beta}, \\
\partial_{t} z_{\alpha} & =D_{\phi} g_{\alpha} \\
& =\sum \frac{\partial g_{\alpha}}{\partial u_{i}} \partial^{i} \phi+\sum \frac{\partial g_{\alpha}}{\partial w_{\beta}} z_{\beta},
\end{aligned}
$$

Let us denote by $X_{\alpha}$ (resp. $\left.Y_{\alpha}\right)$ the right hand side of (4.1 a) (resp. (4.1 b)). We now prove that (4.1) is integrable. First note that

$$
\partial_{t}=\Sigma \partial^{i} F \partial / \partial u_{i}+\sum g_{\alpha} \partial / \partial w_{\alpha}
$$

in $A_{1}$ and hence we have

$$
\begin{aligned}
& {\left[\partial_{t}, \partial / \partial u_{i}\right]=-\sum \frac{\partial}{\partial u_{i}}\left(\partial^{j} F\right) \partial / \partial u_{j}-\sum \frac{\partial g_{\alpha}}{\partial u_{i}} \partial / \partial w_{\alpha},} \\
& {\left[\partial_{t}, \partial / \partial w_{\alpha}\right]=-\sum \frac{\partial g_{\beta}}{\partial w_{\alpha}} \partial / \partial w_{\beta} .}
\end{aligned}
$$

Using the above equations, we have

$$
\begin{aligned}
\partial_{t} X_{\alpha}= & \left\{\left(\partial_{t} \frac{\partial f_{\alpha}}{\partial u_{i}}\right) \partial^{i} \phi+\frac{\partial f_{\alpha}}{\partial u_{i}} \partial_{t} \partial^{i} \phi\right\} \\
& +\left(\partial_{t} \frac{\partial f_{\alpha}}{\partial w_{\beta}}\right) z_{\beta}+\frac{\partial f_{\alpha}}{\partial w_{\beta}}\left(\frac{\partial g_{\beta}}{\partial u_{i}} \partial^{i} \phi+\frac{\partial g_{\beta}}{\partial w_{\gamma}} z_{\gamma}\right) \\
= & \left\{\frac{\partial}{\partial u_{i}}\left(\partial_{t} f_{\alpha}\right)-\frac{\partial}{\partial u_{i}}\left(\partial^{j} F\right) \frac{\partial f_{\alpha}}{\partial u_{j}}-\frac{\partial g_{\beta}}{\partial u_{i}} \frac{\partial f_{\alpha}}{\partial w_{\beta}}\right\} \partial^{i} \phi
\end{aligned}
$$




$$
\begin{aligned}
& +\frac{\partial f_{\alpha}}{\partial u_{i}} \partial_{t} \partial^{i} \phi+\left\{\frac{\partial}{\partial w_{\beta}}\left(\partial_{t} f_{\alpha}\right)-\frac{\partial g_{\gamma}}{\partial w_{\beta}} \frac{\partial f_{\alpha}}{\partial w_{\gamma}}\right\} z_{\beta} \\
& +\frac{\partial f_{\alpha}}{\partial w_{\beta}}\left(\frac{\partial g_{\beta}}{\partial u_{i}} \partial^{i} \phi+\frac{\partial g_{\beta}}{\partial w_{\gamma}} z_{\gamma}\right) \\
= & \left\{\frac{\partial}{\partial u_{i}}\left(\partial_{t} f_{\alpha}\right)-\frac{\partial}{\partial u_{i}}\left(\partial^{j} F\right) \frac{\partial f_{\alpha}}{\partial u_{j}}\right\} \partial^{i} \phi . \\
& +\frac{\partial f_{\alpha}}{\partial u_{i}} \partial_{t} \partial^{i} \phi+\frac{\partial}{\partial w_{\beta}}\left(\partial_{t} f_{\alpha}\right) z_{\beta},
\end{aligned}
$$

where, using the Einstein rule, we omitted the summation sign. Similarly we have

$$
\partial Y_{\alpha}=\frac{\partial}{\partial u_{i}}\left(\partial g_{\alpha}\right) \partial^{i} \phi+\frac{\partial}{\partial w_{\beta}}\left(\partial g_{\alpha}\right) z_{\beta},
$$

where we used the equalities:

$$
\begin{aligned}
& {\left[\partial, \partial / \partial u_{i}\right]=-\partial / \partial u_{i-1}-\frac{\partial f_{\alpha}}{\partial u_{i}} \partial / \partial w_{\alpha}} \\
& {\left[\partial, \partial / \partial w_{\alpha}\right]=-\frac{\partial f_{\beta}}{\partial w_{\alpha}} \partial / \partial w_{\beta} .}
\end{aligned}
$$

By (4.2), (4.3) and $\partial_{t} f_{\alpha}=\partial g_{\alpha}$ we obtain

$$
\begin{aligned}
\partial_{t} X_{\alpha}-\partial Y_{\alpha} & =\frac{\partial f_{\alpha}}{\partial u_{i}}\left\{\partial^{i} \partial_{t} \phi-\frac{\partial}{\partial u_{j}}\left(\partial^{i} F\right) \partial^{j} \phi\right\} \\
& =\frac{\partial f_{\alpha}}{\partial u_{i}}\left(\partial^{i} \partial_{t} \phi-D_{\phi} \partial^{i} F\right) \\
& =\frac{\partial f_{\alpha}}{\partial u_{i}} \partial^{i}\left(\partial_{t} \phi-D_{\phi} F\right) \\
& =0 .
\end{aligned}
$$

Therefore, if the system (4.1) for $z_{\alpha}$ has no solution in $A_{1}$, we can define new pseudopotentials $z_{\alpha}$ by (4.1). This completes the proof. q. e.d.

Using the above theorem repeatedly, we know the following: There exists an extended differential algebra $S_{\infty}=\left\{A_{\infty} ; \partial, \partial_{t}\right\}$ of $S$ such that the derivation $D_{\phi}$ can be extended to a derivation of $A_{\infty}$ commuting with $\partial, \partial_{t}$. (The pseudopotentials constituting $S_{\infty}$ may be infinite.) This is nothing but the non-local symmetry in the sense of [7].

REMARK 1. Note that in general $z_{\alpha}$ is not unique when it can be integrated in $A_{1}$.

REMARK 2. Let us consider the symmmetry of the modified KdV equation. We use the same notations as in $\S 3$, so that $A_{1}$ denotes $C^{\infty}(R \times$ 
$\{w\})$, where $w$ is defined by (3.5). Set $D_{\phi} w=W$, where $\phi=\sin 2 w$. Then it is shown that (3.4) is nothing but the equation (4.1) which must be satisfied by $W$.

\section{$\S 5$. Symmetry of the KdV equation}

In this section we consider only polynomial functions by some technical reasons. Therefore $A$ denotes the space $\boldsymbol{R}\left[x, t, u, u_{1}, \ldots\right]$ of polynomial functions on $R=\left\{\left(x, t, u, u_{1}, \ldots\right)\right\}$.

Let us consider the symmetry $\psi$ defined by (3.9) of the KdV equation. Since $w=w(k)$ depends on $k$, set

$$
w_{i}=\left.\frac{1}{i !} \frac{\partial^{i} w}{\partial k^{i}}\right|_{k=0} .
$$

Therefore $w(k)=\sum_{i=0}^{\infty} w_{i} k^{i}$ holds formally. Similarly set $v=\sum_{i=0}^{\infty} v_{i} k^{i}$. $w_{i}$ and $v_{i}$ are pseudopotentials. The actions of $\partial$ and $\partial_{t}$ to $w_{i}$ (resp. $v_{i}$ ) are determined by the formal expansions of (3.6) (resp. (3.11)) with respect to $k$. For example we have

$$
\partial w_{i}=\sum_{r=0}^{i} w_{r} w_{i-r}
$$

for $i \geqq 2$ and

$$
\partial v_{i}=4 \sum_{r=0}^{i}(r+1) w_{i-r} w_{r+1}-2 \sum_{r=0}^{i} w_{r} v_{i-r}
$$

for $i \geqq 1$. We set $R_{\infty}=R \times\left\{\left(w_{0}, w_{1}, \ldots ; v_{0}, v_{1}, \ldots\right)\right\}$ and denote by $A_{\infty}$ the space of polynomial functions on $R_{\infty}$. Then $S_{\infty}=\left\{A_{\infty} ; \partial, \partial_{t}\right\}$ is an extended differential algebra of $S$.

REMARK. For $f \in A_{\infty}$ let us define the degree of $f$ in such a way that $\operatorname{deg} x=1$, deg $t=3$, deg $u_{i}=i+2$ $\operatorname{deg} w_{i}=1-2 i$ and $\operatorname{deg} v_{i}=-1-2 i$.

If we define $\operatorname{deg} k=2$, then $w(k)$ (resp. $v(k)$ ) is homogeneous of degree 1 (resp. -1 ). From this it is shown that if $\operatorname{deg} f=r$ for $f \in A_{\infty}$, then $\operatorname{deg}(\partial f)=$ $r+1$ and $\operatorname{deg}\left(\partial_{t} f\right)=r+3$.

First we prove

Lemma. For $f \in A_{\infty}$, suppose

$$
\partial f=c w_{0} f,
$$

where $c$ is a constant. Then $f$ depends only on $t$. In particular if $c \neq 0$, then 
$f=0$.

PROOF. Since $\partial u_{i}=u_{i+1}$, it is easy to see that $f$ is independent of $u_{i}^{\prime}$ 's. Similarly $f$ does not depend on $w_{0}$. Next suppose that $f$ is a polynomial of $x, t, w_{1}, \ldots, w_{n}$ and set

$$
f=\sum_{r=0}^{N} a_{r} w_{n}^{r}
$$

where $a_{r}$ is a polynomial independent of $w_{n}$. We will prove the lemma for the above $f$ by induction on $n$. Assume that

$$
\partial f=c w_{0} f .
$$

Since $\partial w_{n}=2 w_{0} w_{n}+$ (the terms independent of $w_{n}$ ) from (5.1), we obtain, by comparing the coefficients of $w_{n}^{N}$ is (5.3), the following equation;

$$
\partial a_{N}+2 N a_{N} w_{0}=c w_{0} a_{N} .
$$

Therefore the induction assumption implies $a_{N}=0$ if $c \neq 2 N$. When $c=2 N$, $a_{N}$ is a polynomial of $t$. In this case, let us consider the coefficient of $w_{n}^{N-1}$ in (5.3). Using $c=2 N$, we have

$$
\partial a_{N-1}-2 a_{N-1} w_{0}+N a_{N}\left(\partial w_{n}-2 w_{0} w_{n}\right)=0 .
$$

Set $a_{N-1}=\sum b_{\alpha} w^{\alpha}$, where $b_{\alpha}$ is independent of $w_{i}$ and $\alpha=\left(\alpha_{1}, \ldots, \alpha_{n-1}\right), w^{\alpha}=$ $w_{1}^{\alpha_{1}} \ldots w_{n-1}^{\alpha_{n-1}}$. Considering the terms containing $w_{0}$ in (5.4), we obtain

$$
\sum 2|\alpha| b_{\alpha} w^{\alpha}-2 \sum b_{\alpha} w^{\alpha}=0,
$$

where $|\alpha|=\alpha_{1}+\ldots+\alpha_{n-1}$. This implies $|\alpha|=1$, that is, $a_{N-1}$ is linear with respect to $w_{i}$. Then it is easy to see that (5.4) implies $a_{N}=a_{N-1}=0$. Therefore we know that the lemma holds for $f$ independent of $v_{i}$ 's.

Finally let us consider the general case. Let $f=f\left(x, t: w_{1}, w_{2}, \ldots\right.$; $\left.v_{0}, \ldots, v_{n}\right)$. Then similar induction arguments on $n$ implies that the lemma holds for general $f$.

q. e. d.

In particular we have

PROPOSITION. $\quad$ ker $\partial \cap$ ker $\partial_{t}=\boldsymbol{R}$.

Let us return to the symmetry $\phi=\psi(k)$. Set $\psi(k)=\sum_{i=0}^{\infty} \phi_{i} k^{i}$. Each $\phi_{i}$ is a symmetry. We want to extend the derivation $D_{\psi_{i}}$ to a derivation $A_{\infty} \longrightarrow A_{\infty}$. First of all we will show the uniqueness of the extension. Set $D_{\psi_{i}} w_{0}=\alpha_{0}$. Then $\alpha_{0}$ satisfies

$$
\partial \alpha_{0}=D_{\psi_{i}} \partial w_{0}
$$




$$
\begin{aligned}
& =D_{\psi_{i}}\left(2 u+w_{0}^{2}\right) \\
& =2 \psi_{i}+2 w_{0} \alpha_{0} .
\end{aligned}
$$

Hence if $\alpha_{0}^{\prime}$ satisfies the same equation, we have

$$
\partial\left(\alpha_{0}-\alpha_{0}^{\prime}\right)=2 w_{0}\left(\alpha_{0}-\alpha_{0}^{\prime}\right) .
$$

Then the lemma implies $\alpha_{0}=\alpha_{0}^{\prime}$. Suppose that the extension $D_{\psi_{i}}: \boldsymbol{R}[x, t ; u$, $\left.u_{1}, \ldots ; w_{0}, \ldots, w_{j}\right] \longrightarrow A_{\infty}$ is unique. Set $D_{\psi_{i}} w_{r}=\alpha_{r}$ for $0 \leqq r \leqq j+1$. We have, from (5.1),

$$
\partial \alpha_{j+1}=2 w_{0} \alpha_{j+1}+\beta,
$$

where $\beta$ is a polynomoal of $w_{r}$ 's and $\alpha_{0}, \ldots, \alpha_{j}$. Therefore we again apply the lemma to prove that $\alpha_{j+1}$ is unique. By similar arguments we can show that the image $D_{\psi_{i}} v_{r}$ of $v_{r}$ is also unique.

Next suppose that the 1-parameter family of derivations $D_{\psi(k)}$ can be extended to a derivation $A_{\infty} \longrightarrow A_{\infty}$ and set

$$
D_{\psi(k)}=\sum k^{i} D_{i} .
$$

Since $\left[\partial, D_{i}\right]=\left[\partial_{t}, D_{i}\right]=0$ and

$$
D_{\psi(k)} u=\psi(k)=\sum \psi_{i} k^{i},
$$

we have $D_{i}=D_{\psi_{i}}$.

Under these preparations we set

$$
\begin{gathered}
\xi=(p-q)\left\{v(p)-w^{\prime}(p)\right\} \widetilde{w}^{2}+\widetilde{w}+v(p)-w^{\prime}(p), \\
\begin{aligned}
\eta=2 \frac{\partial \widetilde{w}}{\partial q}-\frac{\widetilde{w}-w^{\prime}(p)}{p-q} & +\widetilde{w}\left(w^{\prime}(p)-v(p)\right)\left(4 w^{\prime}(q)-2 v(q)-\widetilde{w}\right) \\
& -\frac{v(p)-v(q)}{p-q},
\end{aligned}
\end{gathered}
$$

where $p, q$ are parameters and

$$
\widetilde{w}=\frac{w(p)-w(q)}{p-q} .
$$

Note that $\xi$ and $\eta$ have no singularity at $p=q$. Hence we can set $\xi=$ $\sum \xi_{i j} p^{i} q^{j}$ and $\eta=\sum \eta_{i j} p^{i} q^{j}$. Let us define the derivation $D_{\psi_{i}}$ in $A_{\infty}$ by $D_{\psi(p)} w(q)$ $=\xi$ and $D_{\psi(p)} v(q)=\eta$. In other word, we set $D_{\psi_{i}} w_{j}=\xi_{i j}$ and $D_{\psi_{i}} v_{j}=\eta_{i j}$. (Note that $D_{\psi(p)}=\sum p^{i} D_{\psi_{i}}$ ) Then we can prove by direct calculations that $\xi$ satisfies the following equations;

$$
\left\{\begin{array}{l}
\partial \xi=D_{\psi(p)} \partial w(q) \\
\partial_{t} \xi=D_{\psi(p)} \partial_{t} w(q)
\end{array}\right.
$$


$\eta$ also satisfies similar equations to (5.5). These means that $D_{\psi_{i}}$ commutes with $\partial$ and $\partial_{t}$ in $A_{\infty}$.

For symmetries $\phi, \phi^{\prime}$ let us define the Poisson bracket $\left\{\phi, \phi^{\prime}\right\}$ by

$$
\begin{aligned}
\left\{\phi, \phi^{\prime}\right\} & =\left[D_{\phi}, D_{\phi^{\prime}}\right](u) \\
& =D_{\phi} \phi^{\prime}-D_{\phi^{\prime}} \phi .
\end{aligned}
$$

Then we can prove by direct calculations that $\{\psi(p), \psi(q)\}=0$.

To resume, we have

THEOREM 2. $D_{\psi_{i}}$ can be uniquely extended to a derivation from $A_{\infty}$ to $A_{\infty}$. Moreover $\left\{\psi_{i}, \psi_{j}\right\}=0$ holds for any $i, j$.

Finally we give a remark on the $\mathrm{KdV}$ hierarchy. Let $\phi_{k}=L^{k} u_{1}$, where $L$ is the recursion operator. The totality of the evolution equations $u_{t}=\phi_{k}$ is called the KdV hierarchy. In particular consider the 5 -th order $\mathrm{KdV}$ equation

$$
u_{t}=\phi_{2}
$$

Define a pseudopotential $y$ by

$$
\left\{\begin{aligned}
& y_{x}=2 u+y^{2}-k \\
& y_{t}=4\left(u_{2}+4 k u+4 k^{2}+6 u^{2}\right) y^{2}+4\left(u_{3}+12 u u_{1}+4 k u_{1}\right) y \\
&+2\left(u_{4}+16 u u_{2}+2 k u_{2}+12 u_{1}^{2}+24 u^{3}\right. \\
&\left.+4 k u^{2}+8 k^{2} u-8 k^{3}\right) .
\end{aligned}\right.
$$

It is shown that the transformation $u \longrightarrow \tilde{u}=-u-y^{2}+k$ is also a Bäcklund transformation of (5.6). Note that the right hand of $(5.7 \mathrm{a})$ is identical with the right hand of $(3.6 \mathrm{a})$. This suggest that the transformation $u \longrightarrow$ $\tilde{u}$ represents a symmetry of the KdV hierarchy.

It is well known that the symmetry of the KdV hierarchy is a certain Kac-Moody Lie algebra (for example, see [3]). The relation between the Kac-Moody Lie algebra and the symmetry in this paper seems to be an interesting ploblem.

\section{References}

[1] N. K. IBRAGimOV and R. L. ANDERSON, Lie-Backlund tangent transformations. J. Math. Anal. Appl. 59 (1977), 145-162.

[2] N. K. IBRAGimOv and A. B. ShABAt, Evolution equations with nontrivial Lie-Backlund group, Funkt. Anal. Priloz. 14 (1980), 25-36.

[3] M. JIMBO and T. MIWA, Solitons and infinite dimensional Lie algebras, Publ. RIMS Kyoto Univ. 19 (1983), 943-1001.

[4] Backlund Transformations, edited by R. M. Miura, Lecture notes in Math. 515 (1975), Springer. 
[ 5 ] P. T. Olver, Evolution equations possessing infinitely many symmetries, J. Math. Phys. 18 (1977), 1212

[ 6 ] A. M. VinOGRADOV and I. S. KRASIL'SHCHIK, A method of computing higher symmetries of nonlinear evolution equations, and nonlocal symmetries, Soviet Math. Dok. 22 (1980), 235.

[ 7 ] A. M. VINOGRADOV and I. S. KRASIL'SHCHIK, On the theory of nonlocal symmetries of nonlinear partial differential equations, Soviet Math. Dokl. 29 (1984), 337-341.

[ 8 ] H. D. WAHLQUisT and F. B. EstaBRoOK, Prolongaton structures of nonlinear evolution equations, J. Math. Phys. 16 (1975), 1-7.

Department of Mathematics

Faculty of General Education

Ehime University 